\section{MS25-P6 Two-dimensional oxide quasicrystals from perovskites}

Stefan Förster ${ }^{1}$, Sebastian Schenk ${ }^{1}$, Klaus Meinel ${ }^{1}$, Rene Hammer $^{1}$, Florian Schumann ${ }^{1}$, Wolf Widdra ${ }^{1,2}$

1. Institute of Physics, Martin-Luther-Universität Halle-Wittenberg 2. Max-Planck-Institut für Mikrostrukturphysik

email: stefan.foerster@physik.uni-halle.de

In addition to the well-known intermetallic and soft quasicrystals (QC), recently a two-dimensional oxide quasicrystal (OQC) has been discovered [1]. This OQC is derived from $\mathrm{BaTiO}_{3}$ thin films on a hexagonal $\mathrm{Pt}(111)$ substrate. Low-energy electron diffraction (LEED) reveals a twelve-fold rotational symmetry. Scanning tunneling microscopy (STM) at room temperature as well as at low temperatures $(80 \mathrm{~K})$ allow to resolve the atomic structure. The aperiodic structure is formed by primitive atomic arrangements in squares, triangles, and rhombs with a universal edge length of $0.69 \mathrm{~nm}$. The resulting tiling is comparable to an ideal Stampfli-Gähler tiling [2]. In addition to this dodecagonal atomic arrangement, building blocks of squares, triangles, and rhombs are also found on $(2+\sqrt{3})$ and $(2+\sqrt{3})^{2}$ larger scales indicating the characteristic self-similarity of an ordered QC. The high-resolution STM measurements allow furthermore to identify phason flips on the atomic scale.

The observed interface-driven formation of a $2 \mathrm{D}$ OQC is not limited to this particular materials combination. Following an analogous preparation procedure, we show that $\mathrm{SrTiO}_{3}$ on $\mathrm{Pt}(111)$ develops an OQC as well. As a consequence of the $2 \%$ smaller lattice constant of $\mathrm{SrTiO}_{3}$ in comparison to $\mathrm{BaTiO}_{3}$, the fundamental length of the $\mathrm{SrTiO}_{3}$-derived OQC is $2 \%$ smaller. Nevertheless, the epitaxial alignment of the $\mathrm{SrTiO}_{3}$-derived $\mathrm{OQC}$ with respect to the $\mathrm{Pt}(111)$ lattice as determined by LEED as well as the local atomic arrangement as measured by STM are identical with that of the $\mathrm{BaTiO}_{3}$-derived OQC. Our results suggest that OQC formation is a general process of perovskite oxides on suitable substrates.

[1] S. Förster, K. Meinel, R. Hammer, M. Trautmann, and W. Widdra, Nature 502 (2013) 215.

[2] F. Gähler in: Quasicrystalline Materials (World Scientific, 1988)

This work is supported by the Deutsche Forschungsgemeinschaft (DFG) through SFB 762.

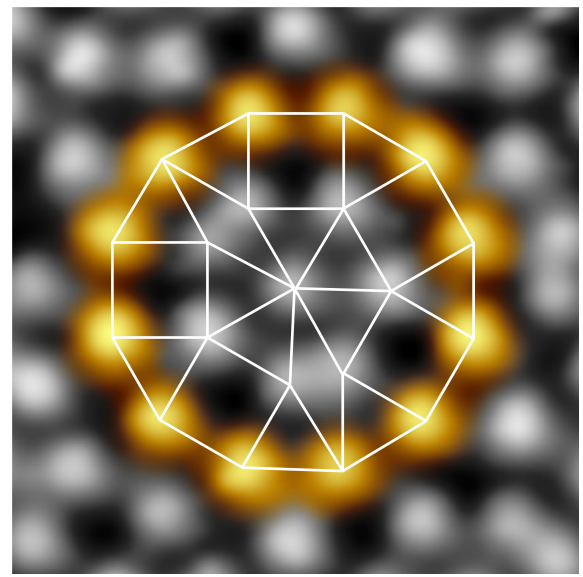

Figure 1. Atomically resolved STM image of the typical

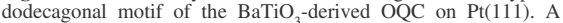
similar tiling is observed for the $\mathrm{SrTiO}_{3}$-derived OQC. $4 \times 4 \mathrm{~nm}^{2}, \mathrm{I}=$ $30 \mathrm{pA}, \mathrm{U}=0.1 \mathrm{~V}$

Keywords: Oxide quasicrystal, Bariumtitanate, Strontiumtitanate, STM, LEED 